\title{
DESARROLLO DE UN MODELO DE PLAN DE MANEJO PARA ÁREAS PROTEGIDAS EN BOSQUES CON ARAUCARIA EN EL SUR DE BRASIL
}

\author{
Maria Augusta Doetzer Rosot ${ }^{1}$, Pablo Cruz ${ }^{2}$, Yeda Maria Malheiros de Oliveira ${ }^{3}$. \\ Hugo Rivera ${ }^{4}$ y Patricia Povoa de Mattos $^{5}$
}

\section{RESUMEN}

El presente trabajo se inserta en el ámbito de convenios de cooperación técnica establecidos entre instituciones de fomento e investigación forestal chilenas y brasileñas, cuyo propósito es el intercambio de tecnologias en las áreas de monitoreamiento ambiental y manejo forestal.

El proyecto objeto de este estudio especifico tiene por finalidad el desarrollo de un modelo de plan de manejo para áreas protegidas en bosques con araucaria presentes en el sur de Brasil, con base en el estudio de caso de un área piloto perteneciente a EMBRAPA (Empresa Brasileña de Investigación Agropecuaria). La reserva forestal EMBRAPA-EPAGRI posee 1.157 ha, se encuentra localizada en el municipio de Caçador, Estado de Santa Catarina, Brasil, y representa uno de los fragmentos forestales en mejor estado de conservación en la región.

La primera fase de los trabajos consistió en la compilación de la base cartográfica y levantamiento del medio biofísico del área para la elaboración de un Sistema de Informaciones Geográficas (SIG), el que contribuirá para el ordenamiento territorial de la Reserva. Se trabajó con layers de vegetación y de los demás usos de suelo (obtenidos por interpretación visual de la imagen IKONOS), hidrografia, altimetria, suelos, pendiente, exposición, Áreas de Preservación Permanente (APP) y Reserva Legal (RL).

Las informaciones de stock maderero fueron colectadas a través de un inventario forestal estratificado en función de clases físicas homogéneas vinculadas a pendiente y exposición. Datos de crecimiento (incremento periódico anual medio - IPA) por estrato fueron obtenidos por el análisis del incremento diamétrico de los últimos 10 años en tarugos de incremento, extraidos de los árboles de las parcelas del inventario.

Las etapas siguientes contemplan un levantamiento faunistico y de la flora con problemas de conservación en el área de la Reserva.

El cruzamiento de todas esas capas de información espacial permitirá atribuir usos o funciones al territorio de la Reserva, utilizando para ello una "Matriz de Compatibilidad de

\footnotetext{
1-Embrapa Florestas - Brasil - augusta@cnpf.embrapa.br

2-Universidad Mayor - Chile - tacora12@yahoo.es

3-Embrapa Florestas - Brasil - yeda@cnpf.embrapa.br

4-CONAF - Chile - rivera harh@yahoo.es

5-Embrapa Florestas - Brasil - povoa@cnpf.embrapa.br
} 
Objetivos" y una metodologia denominada de "Evaluación Multicriterio" (EMC). El propósito de la EMC es investigar un número de alternativas bajo la luz de criterios diferenciados y objetivos en conflicto y, según esa información, generar soluciones compromiso y jerarquizaciones de las alternativas de acuerdo con su grado de atracción.

Se definieron preliminarmente el objetivo principal de la Reserva, que es la investigación de los ecosistemas forestales, y los objetivos especificos, que son la preservación, la educación y capacitación, y la producción maderera y no-maderera. El principal resultado de la EMC, mapa de zonificación de la Reserva, servirá de base para la elaboración del plan de manejo. A partir de este plan serán establecidos diversos programas o acciones a ser aplicados conforme los objetivos especificos definidos para cada zona de la Reserva. Especial énfasis será dado a las áreas destinadas a la producción, pretendiéndose desarrollar e implementar un modelo de ordenamiento forestal para los bosques con araucaria, teniendo por objetivo la generación de productos madereros y no madereros. Se espera de este modo responder a interrogantes formuladas por la legislación ambiental vigente (Decreto 750/1993 y Resolución 278/2001CONAMA) en relación al establecimiento "... de criterios técnicos, cientificamente probados, que garanticen la sustentabilidad de la explotación y conservación genética de las poblaciones explotables".

Al mismo tiempo se procura la definición de un nuevo paradigma para la conservación y uso de los bosques con araucaria, dado que se considera que la legislación, aunque bastante restrictiva en cuanto al aprovechamiento de los recursos madereros, no ha sido eficiente para contener la eliminación de la vegetación natural y la conversión de la tierra para otros usos como agricultura y ganaderia, por ejemplo.

También en el ámbito del proyecto se pretende definir y/o adaptar subtipologias ya existentes de los Bosques con Araucaria que sean más ajustadas con la realidad observada en fragmentos que sufrieron acción antrópica en los más variados niveles.

En una fase posterior se desea establecer e implementar esquemas silviculturales especificos a cada situación, evaluándose sus efectos a lo largo del tiempo por medio de técnicas de monitoreamiento.

Palabras clave: Araucaria angustifolia, Pino Paraná, Manejo Forestal, Áreas Protegidas. 


\section{DEVELOPMENT OF A MANAGEMENT PLAN MODEL FOR PROTECTED AREAS IN SOUTHERN BRAZIL ARAUCARIA FORESTS}

\section{SUMMARY}

This work is part of agreements involving technical cooperation between Brazilian and Chilean educational and research institutions, aiming technologies interchange in environment monitoring and forest management.

This specific study focuses on the development of a management plan model for protected areas in the Araucaria forest in southern Brazil, based on a case study of a pilot area owned by EMBRAPA, the Brazilian Agricultural Research Corporation. EMBRAPA-EPAGRI's Forest Reserve (RFEE) has an area of 1,157 hectares and is located in the Municipality of Caçador, Santa Catarina State. The RFEE is recognized as one of most conserved forest remnants in the Brazilian Mixed Ombrophylous Forest (Araucaria forests).

The first phase of the project consisted of cartographic compilation and biophysical environmental surveys of the area aiming the elaboration of a Geographic Information System (GIS) that will support the Reserve territorial planning. The vegetation layer and other uses of the soil (obtained by visual interpretation of IKONOS imagery), hydrography, altitude, soils, slope, exposition, location of the riparian areas (defined by law as "preservation areas") and Legal Reserve (designed by the Brazilian law as "areas under restricted use") were available from previous work.

Information about wood stock was provided through a forest inventory stratified by homogeneous physical classes considering slope and exposition. Growth data (average periodic annual increment over the last ten years) by stratum was obtained by the diametric increment analysis of increment cores taken from trees located in the inventory plots.

The next stages of the project foresee the endangered flora and fauna surveys.

The cross tabulation of all these spatial information layers will allow to attribute uses or functions to the territory using an "Objective Compatibility Matrix" that is part of the methodology called Multicriteria Evaluation. The purpose of this technique is to search a number of alternatives through differentiated criteria and conflicted objectives and, according to this information, generate solutions, commitments and hierarchization of alternatives, according to their attractiveness.

In a preliminary analysis, the main objective of the Reserve was defined as the research on forest ecosystems and specific objectives included preservation, people education and qualification, and production (timber and no-timber). The main result of the Multicriteria Evaluation, the Reserve Zoning Map, will be used as a base to elaborate the management 
plan. Several programs or actions will be established or applied according to the specific objectives defined to each zone of the Reserve. A special emphasis will be given to the areas destined to production, intending to develop and to implement a forest management model for the Araucaria forest, aiming timber and non-timber products. With this project, one expects to answer questions formulated by the Brazilian environmental statutory law (Acts called Decree 750/1993 and Resolution 278/2001-CONAMA, the Brazilian Environmental Council) concerning the establishment of "... technical criteria, scientifically based, that guarantee the sustainability of the forest exploitation and the genetic conservation of the exploitable populations".

At the same time the project is looking for a new paradigm definition to the conservation and use of the Araucaria forest considering that the legislation, although very restrictive to the exploitation of timber resources, has not been efficient to avoid the suppression of natural vegetation and land conversion to other uses as agriculture and cattle breeding, for example.

The project also aims to define and/or adapt Araucaria forest sub-typologies according to the actual situation observed in remnants that suffered anthropogenic interference in different levels.

In the next phase of the project, some silvicultural protocols, specific to each class, will be established and implemented, and their effects throughout the time will be evaluated by monitoring techniques.

Key words: Araucaria angustifolia, Paraná Pine, Forest Management, Protected Areas. 


\section{INTRODUCCIÓN}

El bioma conocido como Mata o Foresta Atlántica, está presente en 17 provincias a lo largo de la costa brasileña y comprende varias regiones fitoecológicas, incluyendo entre ellas los Bosques con Araucaria que ocurren predominantemente en los tres estados meridionales de Brasil. El patrón histórico de colonización observado desde el Siglo XVI, con el establecimiento de villas y ciudades de norte al sur del litoral, determinó la gradual y sistemática explotación de los bosques, haciendo que la Foresta Atlántica constituya hoy uno de los biomas más amenazados del espacio natural brasileño.

La legislación, aunque bastante restrictiva en cuanto al aprovechamiento de los recursos madereros, rio ha sido eficiente para contener la eliminación de la vegetación natural y la conversión de la tierra para otros usos como agricultura y ganaderia, por ejemplo. Por otro lado, desde el inicio de la década del '90 se encuentran suspendidos los planes de manejo o de ordenamiento forestal desarrollados para predios ubicados en este bioma, hasta que sean respondidas las interrogantes formuladas por la legislación ambiental vigente (Decreto 750/1993 y Resolución 278/2001-CONAMA) en relación al establecimiento "...de criterios técnicos, cientificamente probados, que garanticen la sustentabilidad de la explotación y conservación genética de las poblaciones explotables". Esta es una demanda que se planteó a la comunidad cientifica y que suele ser atendida mediante investigación de largo plazo basada en el estudio de la autoecologia de las especies, de las interacciones fauna-flora, de los factores fisicos del medio (suelos, relieve, agua, luminosidad) y su efecto sobre la producción, de la caracterización de los rodales en términos de estructura y dinámica (Narvaes et al., 2005) y, finalmente, de la relación entre los bosques y la población en términos de percepción de la importancia y de las posibilidades de uso de los recursos naturales.

Actualmente el desarrollo e implementación de planes de manejo están restringidos a la Amazonia y los modelos adoptados para esa región no son aplicables a las condiciones boscosas presentes en otros biomas. La carencia de estudios silviculturales y de manejo para los bosques nativos del sur de Brasil fue lo que motivó el establecimiento del presente proyecto. En la búsqueda de antecedentes relacionados al tema, se constató que existen amplias similitudes entre el "Bosque con Araucaria" brasileño y el "Bosque Siempreverde" chileno, corroboradas por opiniones técnicas de investigadores de ambos paises. Dada la experiencia chilena en el manejo de bosques nativos, se establecieron convenios de cooperación técnica entre instituciones de fomento e investigación forestal chilenas y brasileñas, cuyo propósito es el intercambio de tecnologias en las áreas de monitoreamiento ambiental y manejo forestal.

En el ámbito de estos convenios se inserta el presente proyecto, donde se propondrá un método de manejo para el Bosque con Araucaria, de forma de garantizar su sustentabilidad, esto es, donde se generen productos forestales con fines comerciales, se conserve la base productiva y se consideren los beneficios sociales del bosque, principalmente con el empleo en las actividades silviculturales. Además, se plantea un cambio de paradigma con respecto a áreas protegidas, donde tradicionalmente es vedada toda y cualquier intervención, determinando fuerte tendencia a la preservación, que no siempre está basada en una evaluación técnica o con criterios claros respecto de las condiciones y necesidades del bosque. 


\section{MATERIALES}

\section{Área de Estudio}

La región con Bosques con Araucaria ocupaba originalmente un área de 20 millones de hectáreas de los cuales se estima que existen hoy apenas 400.000 ha (Guerra et al., 2000). En su mayoria estos bosques se presentan en malas condiciones, empobrecidos debido a floreos ejecutados en los últimos 60 años. No es distinta la situación en el predio público donde se desarrolla el proyecto, una reserva forestal (Figura $N^{*} 1$ ) perteneciente a EMBRAPA. La reserva posee 1.157 ha, se encuentra localizada en el Municipio de Caçador, Estado de Santa Catarina, Brasil, y representa uno de los fragmentos forestales en mejor estado de conservación en la región aunque haya sufrido cortas selectivas hasta el inicio de los años ' 80 . La mayor parte de la reserva está cubierta por la tipologia "Foresta Ombrófila Mixta Montana", donde están presentes algunas especies consideradas en riesgo de extinción como Araucaria angustifolia (pino-paraná), Ocotea porosa (imbuia), Ocotea pretiosa (canela-sasafraz) y Ocotea catharinensis (canela-preta). La altitud varia de 1.000 a $1.100 \mathrm{~m}$ y el clima es del tipo Cfb, según la clasificación de Koeppen, presentando veranos calientes e inviernos frios con ocurrencia de heladas. Las clases de suelo Cambisolos + Neosolos son predominantes y el relieve varia de suave ondulado a ondulado. Dos riachuelos y nacientes asociadas caracterizan la hidrografia.

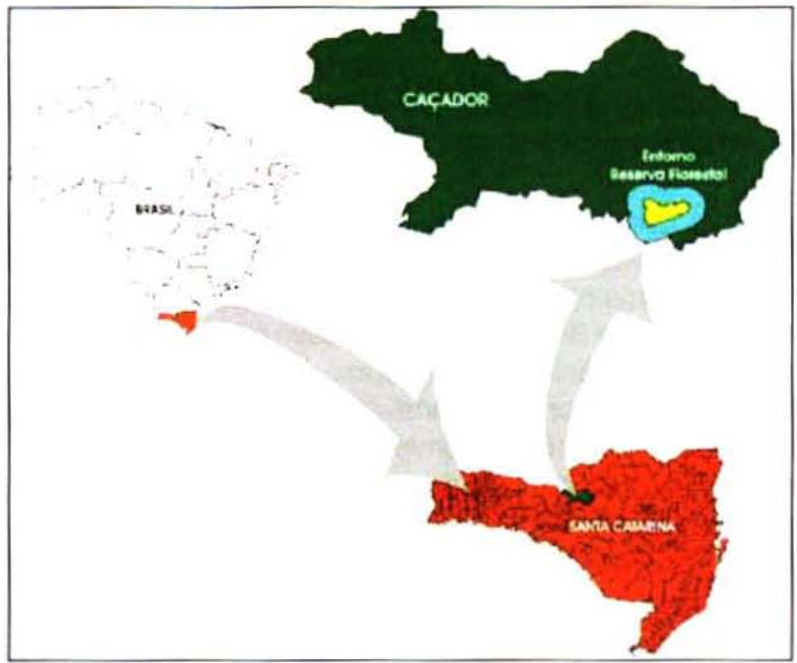

(Fuente: Kurasz, 2005)

\section{Figura $\mathrm{N}^{\circ} 1$ \\ UBICACIÓN DE LA RESERVA FORESTAL EMBRAPAEPAGRI}

Dos planes de manejo fueron desarrollados en los años de 1994 y 1997, pero ninguno de ellos fue implementado, porque se dudaba de su eficacia en proteger el área. A partir de 1998 el predio pasó a denominarse Reserva Forestal EMBRAPAVPAGRI y desde entonces sólo se 
realizan actividades de mantención de caminos y cortafuegos, además de investigaciones tanto en el bosque nativo como en los ensayos de frutales presentes en una pequeña superficie de la reserva.

Araucaria angustifolia o pino-paraná (uno de los dos géneros de coniferas nativos de Brasil) es la especie dominante en el Bosque con Araucaria y fue durante muchas décadas la principal especie maderera explotada en el sur. Hoy tiene su manejo prohibido por ley y es todavia poco lo que se conoce de ella en términos silviculturales. De excepcional forma y buen crecimiento (puede llegar hasta $15 \mathrm{~m}^{3} / \mathrm{ha} / \mathrm{año}$ ), la especie merece que se la estudie más detalladamente pues representa la más rentable alternativa del bosque, tanto por su madera como por los piñones (semillas) que produce, los cuales son tradicionalmente comercializados como alimento.

Lo que se observa, de manera general, es la regeneración deficiente del pino-paraná dentro del bosque por ser una especie exigente en luz. Aunque se esperaria que los disturbios, naturales o provocados por acciones antrópicas, desarrollasen el proceso de establecimiento de nuevas plántulas por ocasión de la abertura del dosel, esto no ocurre. Uno de los motivos para ello puede ser la presencia masiva de la taquara, un tipo de bambú de los géneros Merostachys y Chusquea, que tuvo su establecimiento favorecido por sucesivas intervenciones antrópicas. Por otro lado, se sugiere también que los problemas con la regeneración se deban a acciones predatorias de la fauna que se alimenta de los piñones.

\section{METODOLOGIA}

El modelo de plan de manejo que se pretende desarrollar para la reserva se apoya en tres vertientes distintas:

Ordenación territorial

Ordenación forestal

Actividades de monitoreo

La ordenación territorial (OT) es un diseño de planificación que tiene como objetivo realizar planificación integral, es decir, considerando todos los recursos y limitaciones que cada predio pudiera tener. De esta OT resultará la superficie destinada a manejo forestal, por ejemplo, una vez que se hayan sido analizadas todas las funciones que pudiese desempeñar la cobertura boscosa. El carácter integral que tiene este proceso obliga a levantar una linea base diversa y su esencia es la toma de decisiones sobre el territorio, con toda la gama de informaciones que posee cada área. La base de las actividades realizadas en esta etapa es el Sistema de Informaciones Geográficas (SIG).

La segunda vertiente que prevé un modelo integral de manejo forestal sustentable debe contener los parámetros que definen un sistema silvicultural aplicable a estos bosques. En la ordenación forestal (OF) se emplean los conceptos de enfoque predial, equilibrio de producción y rendimiento sostenido (Rothermel, 2002). Las actividades de monitoreo siguen a la implementación del modelo, mediante la instalación, medición y evaluación de ensayos silviculturales en las superficies bajo manejo. 


\section{Plan de Ordenación Territorial (POT)}

La primera fase de los trabajos consistió en la compilación de la base cartográfica y levantamiento del medio biofisico del área para la elaboración de un Sistema de Informaciones Geográficas (SIG).

La base cartográfica provino de una carta elaborada por la Dirección de Servicios Generales del Ejército (DSG), escala 1:50.000, con curvas de nivel de 20 en 20 metros y puntos con información altimétrica. El mapa en medio analógico fue escaneado, georreferenciado y la red viaria, la hidrografia y las curvas fueron vectorizadas para generar un modelo digital de elevación (MDE) y luego las capas de altimetria, pendiente y exposición.

El levantamiento del medio biofísico incluyó:

Levantamiento semi-detallado de suelos a una escala 1:25.000 con análisis de parámetros físico-quimicos.

Levantamiento de la vegetación en terreno y por medio de técnicas de teledetección usando fotointerpretación en pantalla de una imagen IKONOS multiespectral con resolución de 4 metros.

Informaciones dasométricas colectadas a través de un inventario forestal estratificado en función de clases fisicas homogéneas vinculadas a pendiente y exposición.

Datos de crecimiento (incremento periódico anual medio - IPA) por estrato obtenidos por el análisis del incremento diamétrico de los últimos 10 años de tarugos de incremento, extraidos de los árboles de las parcelas del inventario.

Identificación botánica de todos los árboles muestreados en las parcelas.

En función de la ubicación de los cursos de agua y nacientes fue generada una capa de restricciones legales relativas a las áreas de preservación permanente, asi como se asignó 20 $\%$ de la superficie como "reserva legal" que, según el código forestal brasileño, no puede ser sometida a tala rasa.

Las etapas siguientes prevén un levantamiento de fauna y de la flora con problemas de conservación en el área de la Reserva. El cruzamiento de todas esas capas de información espacial permitirá atribuir usos o funciones al territorio de la Reserva, utilizando para ello una "Matriz de Compatibilidad de Objetivos" y una metodologia denominada de "Evaluación Multicriterio" (EMC). El propósito de la EMC es investigar un número de alternativas bajo la luz de criterios diferenciados y objetivos en conflicto y, según esa información, generar soluciones compromiso y jerarquizaciones de las alternativas de acuerdo con su grado de atracción.

El objetivo principal de la Reserva es posibilitar la investigación de los ecosistemas forestales en distintos niveles, incluyendo los objetivos especificos de preservación, educación y capacitación, y producción (maderera y no-maderera). 
El proceso de asignación de funciones al territorio permite que se alcancen compromisos para una planificación de mediano plazo. El principal resultado de la EMC, mapa de zonificación de la Reserva, servirá de base para la elaboración del plan de manejo. A partir de este plan serán establecidos diversos programas o acciones a ser aplicados conforme los objetivos especificos definidos para cada zona de la Reserva. Específicamente para las superficies asignadas como "zonas de aprovechamiento maderero" se elaborará un plan de ordenación forestal.

\section{Plan de Ordenación Forestal (POF)}

\section{- Modelo del Plan de Manejo Forestal}

El aspecto central de esta etapa del proyecto es crear propuestas silviculturales para las diferentes situaciones que presentan los Bosques con Araucaria, en un formato demostrativo. Se hará énfasis en los detalles prácticos, los costos y rendimiento involucrados y el rendimiento volumétrico en cada caso. Por esta razón es fundamental la elaboración de una tipologia forestal con un sentido silvicultural, es decir, que tenga como objetivo, clasificar los bosques según el tipo de silvicultura posible.

Una componente fundamental es el diseño y construcción de áreas demostrativas, en que sean aplicadas las actividades según su tipologia. Por esta razón, la última fase del proyecto consistirá en la implementación de áreas demostrativas en que se apliquen las propuestas del modelo silvicola.

EI POF se estructurará conforme las siguientes etapas:

Análisis y recopilación de antecedentes (tipologia y silvicultura). La propuesta de manejo se elaborará sobre la base de tres fuentes de información:

Documentación desarrollada en Brasil relativa a la caracterización del bosque, sobre la cual se basará la tipología silvicola.

Documentación de experiencias silviculturales para este bosque, realizadas en Brasil.

Documentación de silvicultura del Tipo Forestal Diempreverde de Chile que pueda ser aplicada al Bosque con Araucaria.

Elaboración de la tipologia silvícola. Dado que las propuestas silvicolas se elaborarán para la clasificacion de las diversas situaciones que presente el bosque, es necesario sistematizar las actuales tipologias con el objeto de agrupar aquellas situaciones con silvicultura común. Asi, la nueva clasificación tendrá un sustento en los actuales trabajos de descripción del bosque. La elaboración deberá tener una fase de validación, consistente en la visita a bosques de la reserva en las que se contrastará cada tipo propuesto con los bosques que representa. En aquellos casos en que sea posible verificar en terreno los resultados de alguna de las actuales clasificaciones, se realizará una visita de ajuste para mejorar la consistencia de los datos. 
Análisis y adaptación de técnicas silvicolas. Se analizará el tipo de silvicultura posible de aplicar a cada una de las tipologias confeccionadas en la etapa anterior. Dado que la proposición de intervenciones, sus objetivos, intensidad, momento de ejecución, y demás indicaciones deben provenir de la experiencia en el manejo del bosque específico al cual se está aplicando la actividad, la base de análisis será la experiencia documentada en Brasil referida a actividades silvicolas en el Bosque con Araucaria. La información descrita será complementada con la experiencia de ensayos silvicolas de Chile, en aquellos casos en que exista una directa similitud entre los tipos de bosque y las respuestas documentadas en ese pais, estén sólidamente probadas en sus resultados y efectos ambientales. Tanto las experiencias brasileñas como las chilenas serán validadas en su consistencia, por la via de visitas a terreno. De esta etapa se tendrá como resultados un manual de actividades silvicolas propuestas para cada una de las categorias descritas en la tipologia silvicultural.

Ensayos silviculturales complementarios. Se levantarán ensayos de las técnicas silvicolas más significativas de la propuesta. Estos ensayos tendrán un carácter demostrativo y de evaluación y seguimiento al efecto esperado en el bosque. Serán evaluados por mediciones anuales que entregarán informaciones sobre la dinámica de crecimiento y producción del bosque después de la aplicación de las técnicas silvicolas propuestas. Además, se desarrollará un estudio de ajuste de la silvicultura a los crecimientos de las especies más importantes por su uso maderero, a través de análisis de fuste. De estos estudios se espera obtener, entre otras informaciones, los momentos de aplicación de las cortas, funciones de crecimiento en altura y funciones de crecimiento volumétrico.

En la primera fase del POF se utilizarán también los datos de incremento periódico anual medio para cálculo de las tasas de aprovechamiento. Con los resultados descritos, se elaborará un modelo de manejo forestal para los Bosques con Araucaria, en formato de guia para silvicultores, que contendrá la identificación de tipologias silvicolas prediales, la definición de esquemas silviculturales y una guia de elaboración de plan de manejo predial.

Las àreas demostrativas tendrán como función la implementación de las actividades silvicolas y su seguimiento posterior, en un formato demostrativo a los diferentes actores del sector forestal. Un aspecto importante de la implementación de silvicultura en las áreas es la aplicación a escala operativa, en la que se deberá resolver el conjunto de faenas indirectas relacionadas con la actividad forestal en bosque nativo, como es la planificación de caminos y vias de saca, dimensionamiento de las actividades anuales en un régimen de rendimiento sostenido, entre otras.

\section{- Elaboración e implementación del POF}

El Plan de Ordenación Territorial (POT) considera las diferentes funciones atribuidas al territorio según la metodologia de evaluación multicriterio, incluyéndose la superficie de aprovechamiento maderero. Se elaborará un Plan de Ordenación Forestal (POF) para la totalidad de dicha superficie. 
El plan estará basado en la estimación de parámetros de Ordenación calculados a partir de información colectada en terreno. Como resultado final, se planificarán las actividades y faenas espacial y temporalmente por un periodo de tiempo determinado. El POF se constituirá de las siguientes fases:

Recopilación POT. Se recopilará, en formato analógico y digital, los mapas de ordenación territorial que incluyen la superficie final considerada como de aprovechamiento maderero. Los trabajos cartográficos subsecuentes, ejecutados en ambiente de SIG, serán elaborados con base en el límite de dicha superficie.

\section{Caracterización cartográfica}

- Levantamiento de información temática con uso de Aeromodelo: La Reserva será sobrevolada con un Aeromodelo desarrollado por EMBRAPA que utiliza un censor digital en el espectro visible e infrarrojo con el objetivo de refinar las tipologias definidas en el POT. Las fotos obtenidas con superposición longitudinal y lateral serán empleadas en la elaboración de una carta temática de tipologias forestales y además se probará algoritmos clasificadores automáticos.

- Elaboración Cartografia final: Se elaborarán coberturas temáticas detalladas para el área de aprovechamiento maderero que contendrán:

Rodales y su fotointerpretación

Infraestructura y caminos

Hidrografia detallada

Mapa de Productividad (compartimentos homogéneos en términos de factores biofisicos: tipologia forestal, exposición y pendiente)

Levantamiento de la información de terreno. Caracterización de rodales: Cada rodal será evaluado desde la perspectiva de su potencial para la producción maderera. Por ello, la primera evaluación será cualitativa, destinada a evaluar la estructura, composición, estado de desarrollo, especies de valor para el manejo, método silvicola para lograr el objetivo, y actividad silvicola de los próximos 10 años, opciones de enriquecimiento, y otros datos de relevancia para la ordenación y silvicultura. Para esta evaluación se utilizará la metodologia de Cruz y Rothermel (1998), usando un formulario estructurado que se aplicará a la condición completa del rodal. Una vez caracterizado el rodal, se realizará un segundo inventario para estimar variables dasométricas y rendimiento volumétrico de las intervenciones silvicolas propuestas en la etapa anterior.

\section{-Elaboración plan de ordenación forestal}

- Procesamiento de la Información predial: Se procesarán los datos y se integrarán las propuestas silvicolas realizadas en terreno. La información cualitativa y de inventario permitirá determinar las siguientes variables de relevancia (las principales) para cada rodal:

Tipo de bosque: se elaborará una caracterización detallada de cada 
situación orientada a clasificar según la tipología propuesta en este proyecto.

Estructura y composición.

Objetivos posibles de manejo y calidad de productos potenciales.

Método silvicola recomendado y actividad silvicola para los próximos 10 años.

Stock estimado de madera en pie.

Intensidad resultante de la aplicación de la actividad silvicola propuesta.

- Cálculo de parámetros de ordenación forestal: Los parámetros de ordenación se utilizan para guiar la planificación cuando ésta se condiciona a una producción bajo rendimiento sostenido. Los parámetros se estimarán con la metodologia de Cruz et al. (2005) que involucra aspectos tales como la tasa de aprovechamiento y el equilibrio de producción. Para esta etapa se considera una evaluación de las funciones de biomasa y volumen existentes, asi como la identificación de software posibles de usar como apoyo a la planificación.

- Elaboración del Plan Operativo Predial para el Periodo: Se realizará la planificación temporal y espacial de las actividades, sujetas a las restricciones dadas por los parámetros de Ordenación Forestal y los objetivos propuestos, para un periodo de 5 años. El plan contendrá los rodales a intervenir asi como las superficies y el volumen estimado de extracción (por año, periodo, predio y por rodal). Para apoyar estas actividades se elaborará cartografia operativa con base en el SIG.

\section{- Ejecución del Plan Operativo}

Definidas las actividades para el período de 5 años, se comenzará su implementación siguiendo las indicaciones del Plan Operativo. Evidentemente, no es posible definir la cantidad y detalle de esas actividades en la etapa de preparación en que se encuentra este proyecto, sin embargo, es posible indicar las temáticas que se considerarán en dicha ejecución.

Las experiencias similares implementadas en Chile indican que las superficies que se deben intervenir en un área bajo rendimiento sostenido fluctúan entre 15 y $35 \%$ de la superficie total de bosque. Para el caso de la Reserva de Caçador podria considerarse una superficie intervenida de 150 a 350 hectáreas (esta superficie se definirá después de la elaboración del POT). Asi, la implementación del plan se hará según las siguientes actividades:

Gestión y preparación de infraestructura

Implementación de faenas o actividades silvicolas directas

Gestión de comercialización

Monitoreo del plan. 


\section{RESULTADOS Y DISCUSIÓN}

\section{SIG y Ordenamiento Territorial de la Reserva}

El levantamiento de suelos para la Reserva permitió identificar nueve unidades de mapeamiento, distribuidas en cuatro órdenes: Cambisolos, Gleisolos, Nitosolos y Neosolos. Mayores detalles pueden ser encontrados en Kurasz et al. (2004). En la Figura $\mathrm{N}^{\circ} 2$ se presenta el mapa de suelos resultante del levantamiento ejecutado.

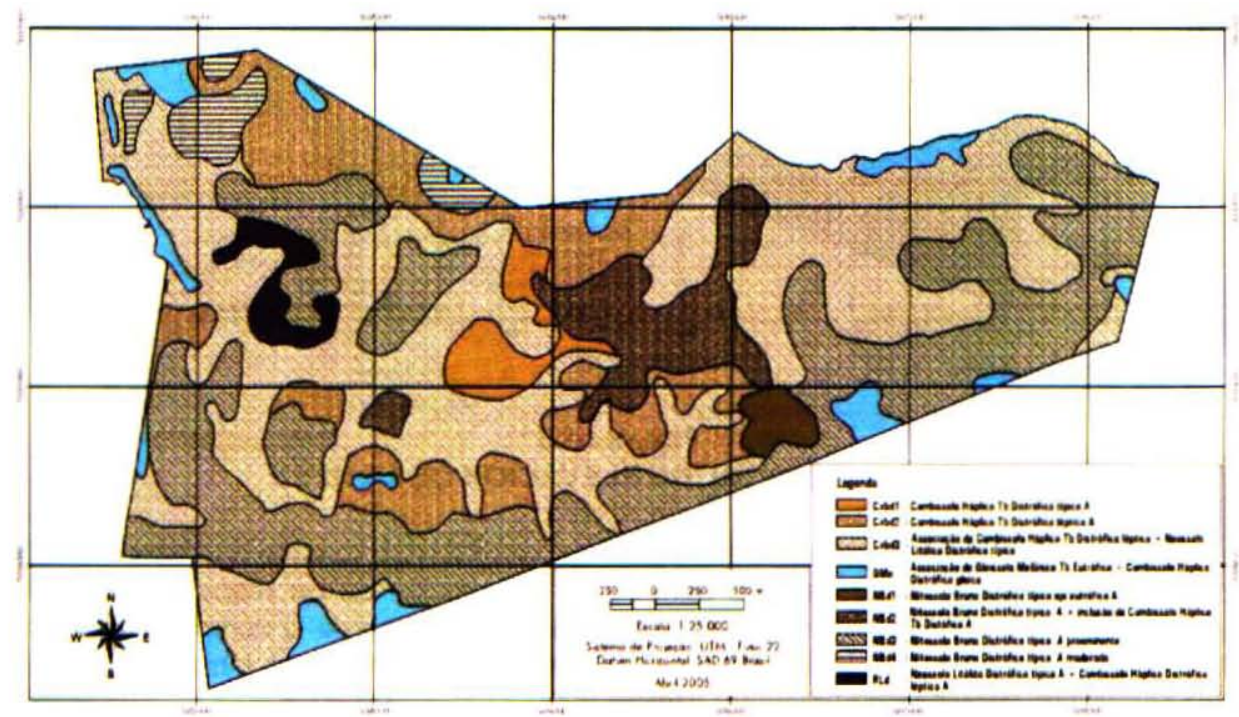

(Fuente: Kurasz, 2005)

\section{Figura $\mathrm{N}^{\circ} 2$}

\section{SUELOS EN LA RESERVA FORESTAL EMBRAPA/EPAGRI}

El modelo digital de elevación (MDE) fue creado con base en un TIN (Triangular Irregular Network), considerando las curvas de nivel y los puntos de altitud. El mapa hipsométrico generado (Figura $\mathrm{N}^{\circ}$ 3) muestra una variación de altitud 920 a $1.075 \mathrm{~m}$. A partir de ello fueron generadas capas de pendiente y exposición, con tres y cuatro clases, respectivamente. La mayor parte de la superficie de la Reserva se encuentra en las clases de pendiente plana $(0-0,5 \%)$ y moderada $(8-20 \%)$, ocupando 34 y $36 \%$ del territorio, respectivamente, lo que determina un relieve ondulado y con exposiciones plana (34\%), norte $(24 \%)$ y oeste $(19 \%)$ de manera predominante (Rivera, 2006). El cruce de estas dos capas tornó posible la generación de 13 clases de medio fisico, utilizadas como estrato en el inventario forestal. En esta fase se optó por no incluir las clases de suelo en la estratificación debido a su escala de levantamiento (1:25.000) y, por otro lado, por la necesidad de investigar más detalladamente la relación suelo-productividad en ensayos localizados y con control más efectivo sobre las fuentes de variación. 


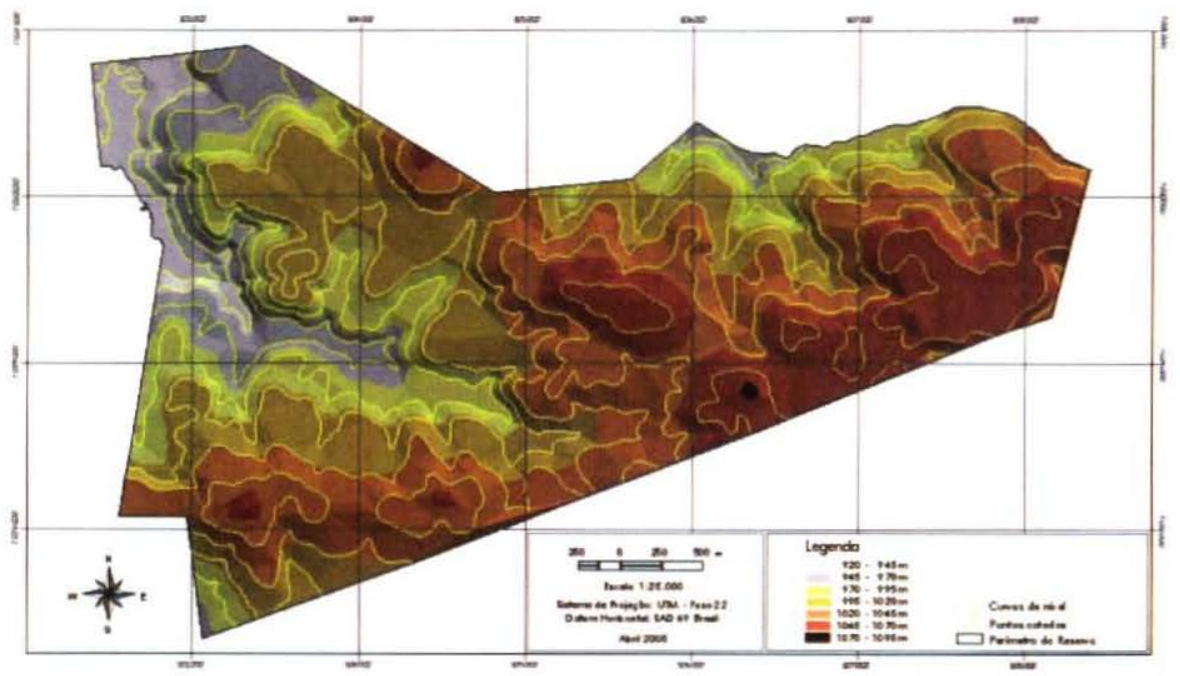

(Fuente: Kurasz, 2005)

\section{Figura $\mathrm{N}^{\circ} 3$ \\ MODELO DIGITAL DE ELEVACIÓN DE LA RFEE}

La fotointerpretación en pantalla de una imagen IKONOS tomada en Febrero de 2004 asociada al levantamiento en terreno permitió la elaboración de un mapa de vegetación, cuyas clases se definieron en función del estado de desarrollo y de la composición floristica del bosque (Figura $\mathrm{N}^{\circ} 4$ ). En un primer nivel se agrupó la vegetación en términos de estados sucesionales (inicial, medio y avanzado) y luego, dentro de cada uno de ellos, en clases según la composición de especies. 

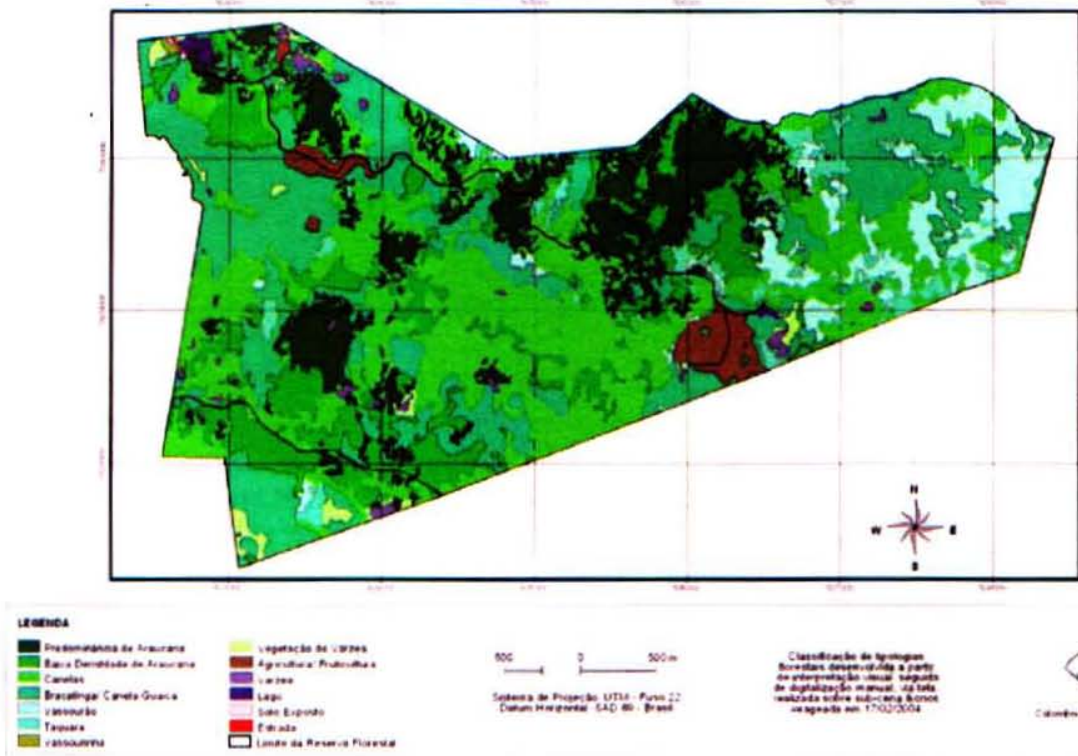

(Fuente: Dlugosz, 2005)

\section{Figura $\mathrm{N}^{\circ} 4$}

\section{MAPA DE USO DEL SUELO EN LA RESERVA FLORESTAL EMBRAPAEPAGRI}

El primer abordaje de evaluación forestal hecho en la Reserva empezó el año 2003 con la recuperación de 10 parcelas permanentes de dimensiones $25 \times 100 \mathrm{~m}$, instaladas en 1989 en el estrato con predominancia de araucaria y que no habian sido remedidas en los años subsecuentes. Se estableció asi el Inventario Forestal Continuo (IFC), demarcándose fisicamente las parcelas con la distribución señalada en la Figura $\mathrm{N}^{\circ} 5$ y midiéndose todos los individuos arbóreos con DAP superior a $20 \mathrm{~cm}$. Cada árbol recibió una etiqueta de identificación y fue pintado a la altura del DAP donde fue medida su circunferencia (CAP). También se midió la altura comercial y se hizo una evaluación de la calidad de la madera a cada árbol. En el procesamiento de este primer inventario se constató la existencia de más de $\mathbf{2 0 0}$ individuos con DAP superior a $20 \mathrm{~cm}$ por hectárea. Mantenciones y remediciones fueran realizadas en el 2004,2005 y 2006 . Hoy estas parcelas ya están integradas a la recién creada Red de Parcelas Permanentes de la Mata Atlántica y Pampa. 


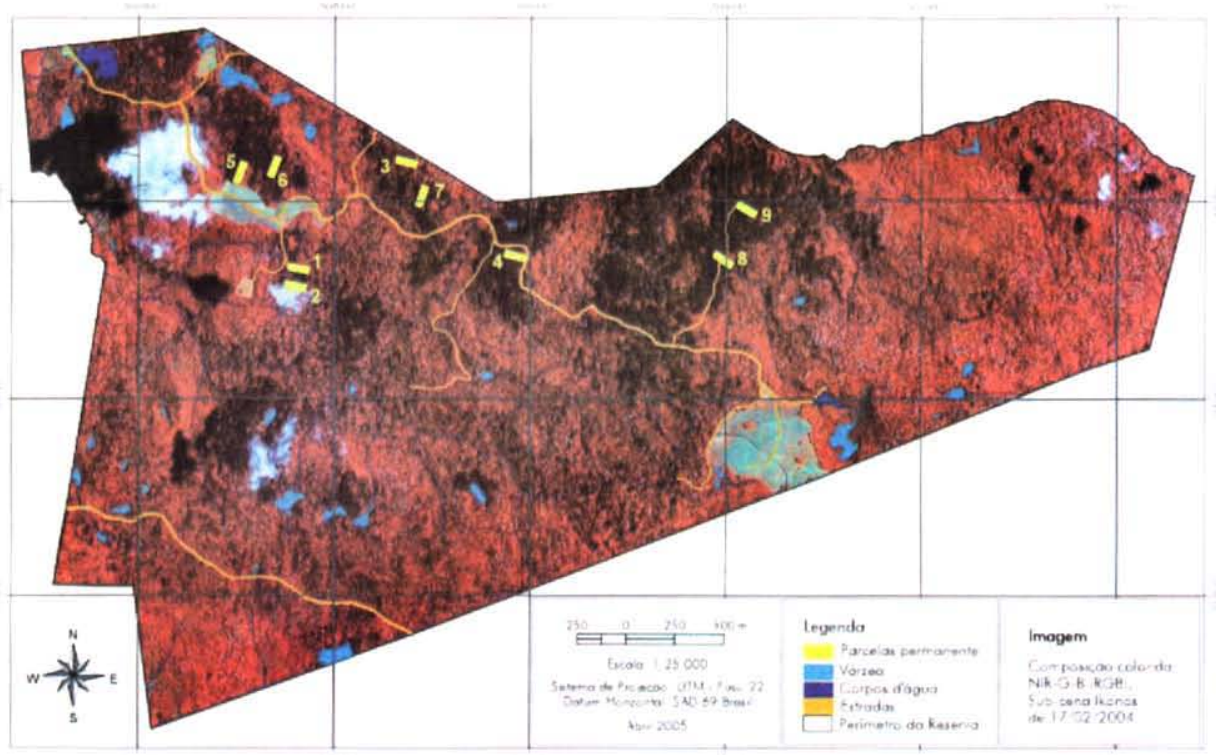

(Fuente: Kurasz, 2005)

Figura $\mathrm{N}^{\circ} 5$

LOCALIZACIÓN DE PARCELAS PERMANENTES EN LA RFEE SEÑALADA SOBRE UNA IMAGEN IKONOS

En el año 2006 se ejecutó un inventario forestal, realizándose 72 parcelas temporales (tres para cada clase de medio físico como mínimo), considerándose $15 \%$ de error de muestreo y $49 \%$ en el coeficiente de variación en relación al área basal, procurándose muestrear todas las clases del medio fisico de forma uniforme en toda la reserva y todos los tipos de vegetación. El área basal promedio fue equivalente a $33,37 \mathrm{~m}^{2} /$ ha (Rivera, 2006), midiéndose todos los árboles con diámetros iguales o mayores que $10 \mathrm{~cm}$. En la etapa siguiente se hará un análisis fitosociológico para estimar abundancia, frecuencia, dominancia y un indice de valor de importancia.

Se realizó la identificación botánica del material arbóreo colectado en las parcelas del inventario, y se confeccionó un herbario que estará localizado en la reserva y que deberá acrecentarse con material de nuevas colectas. Las familias más frecuentes fueron Sapindaceae, Lauraceae, Flacourtiaceae y Myrtaceae, siendo las especies más comúnmente encontradas Cupania vernalis (Cuvatã), Capsicodendron dinisii (Pimenteira), Araucaria angustifolia (Araucaria), y Prunus brasiliensis (Pessegueiro-bravo) de las familias Sapindaceae, Canellaceae, Araucariaceae y Rosaceae, respectivamente (Rivera, 2006). Serán consultados especialistas para una evaluación expedita sobre la presencia de especies de la flora con problemas de conservación y, en caso positivo, con la respectiva espacialización de estos eventos se hará su inserción en el SIG, constituyendo también una capa limitante en la EMC. 
En cada parcela fueron colectados tarugos de cinco árboles, en distintas clases de tamaño para estimar el respectivo incremento periódico anual medio y que a la fecha continúan siendo medidos. Además de investigar correlaciones entre el crecimiento y las variables físicas, se pretende hacer una interpolación con base en los valores puntuales encontrados para cada parcela y asi generar una nueva capa de información para utilizar en la evaluación multicriterio (EMC).

La intersección de las clases de uso del suelo y las superficies definidas como Área de Preservación Permanente (APP) permitió verificar que efectivamente 296 ha contienen vegetación nativa, restando solamente 5 ha como superficies a restaurar, lo que representa una excepción en la propiedad rural de manera general en el sur de Brasil. En este caso especifico, los bajos valores de APP a restaurar se deben al hecho de que esta es un área protegida y que no ha sufrido alteraciones significativas de origen antrópica en los últimos 20 años. Las superficies destinadas a la Reserva Legal propia son equivalentes a 248 ha, o cerca de $21 \%$ de la superficie total. Estos predios suelen utilizarse como zonas de investigación en manejo forestal, exceptuándose el régimen de tala rasa, no permitido por ley. El mapa de restricciones legales (Figura $\mathrm{N}^{\circ} 6$ ) será usado como capa limitante en la EMC.

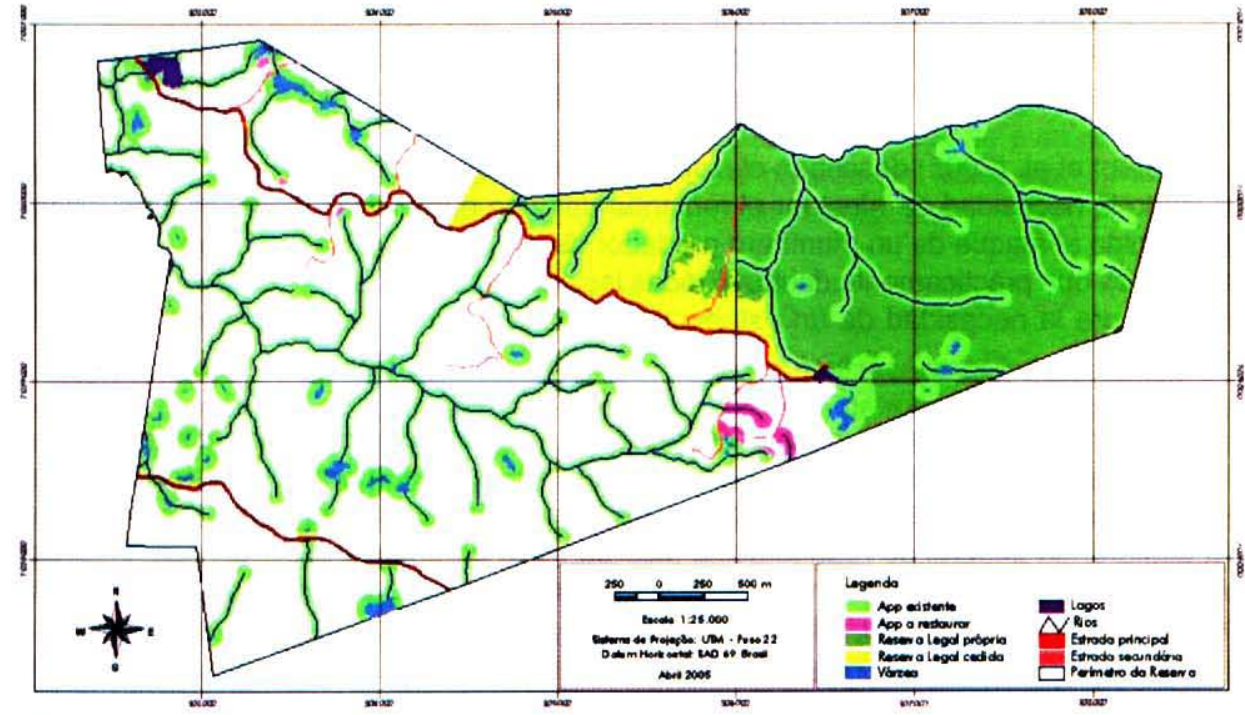

(Fuente: Kurasz, 2005)

Figura $N^{\circ} 6$

RESTRICCIONES LEGALES DE USO EN LA RFEE

Estando disponibles en el SIG todas las capas necesarias, el primer paso será rasterizar las capas de información para permitir la aplicación de álgebra de mapas. La evaluación multicriterio prevé la definición de limitantes de carácter legal (por ejemplo, APP, RL, especies vulnerables de flora y fauna) o ambiental (suelos susceptibles a la erosión), que son variables 
dicotómicas, o sea, se permite (1) o no se permite (0) realizar alguna actividad en alguna superficie. Por otro lado, se definen también los factores, expresados en gradientes, y que pueden ser ambientales, tal como las clases de pendientes y de estadios sucesionales de la vegetación, o económicos, como la accesibilidad a caminos, calidad de la madera en pie, stock y tasa de crecimiento, por ejemplo. El mapa final conteniendo la asignación de funciones al territorio de la Reserva será obtenido con base en la combinación de los factores ambientales y económicos según criterios establecidos en la matriz de compatibilidad de objetivos, mencionada anteriormente, $y$ también considerando la capa de limitantes.

\section{ACTIVIDADES DE MONITOREO}

Las principales acciones relativas a monitoreo serán establecidas después de la planificación y ejecución de un plan de manejo forestal para las superficies a las cuales fueron asignadas estas funciones en el ordenamiento territorial.

En el año 2004 un incendio provocado por una quema en áreas agricolas vecinas alcanzó una pequeña superficie de la Reserva. Para recuperar la cobertura forestal en este lugar, se planteó establecer un ensayo silvicultural de recuperación de área alterada por fuego utilizando plantación en filas, con espaciamiento de $5 \times 5 \mathrm{~m}$, empleándose dos especies nativas que ocurren naturalmente en la Reserva, Araucaria angustifolia (pino-paraná), con semillas, y Ocotea porosa (imbuia), con plántulas, ambas consideradas como vulnerables a la extinción.

Rosot et al. (2005) describen el diseño (Figura $\mathrm{N}^{\circ} 7$ ) y primeros resultados del ensayo, resaltando la necesidad de efectuar el replantio del pino-paraná con plántulas producidas en vivero debido al ataque de un mamifero predador de los piñones, el mono-clavo (Cebus apella var. nigritus), que prácticamente destruyó todas las plántulas que habian nacido de las semillas. Eso corrobora la necesidad de un estudio más detallado de la fauna local y de sus hábitos alimentarios.

En la fase actual, este ensayo viene siendo monitoreado dos veces al año respecto a la supervivencia y adaptación de las plántulas. La próxima etapa prevé una evaluación de la regeneración natural de especies arbóreas de importancia comercial y del comportamiento de la taquara, en general de regeneración muy agresiva en presencia de luz. Los datos están organizados en el SIG para permitir espacialización, control de la condición de la plántula y comparación de parámetros a lo largo del tiempo. 


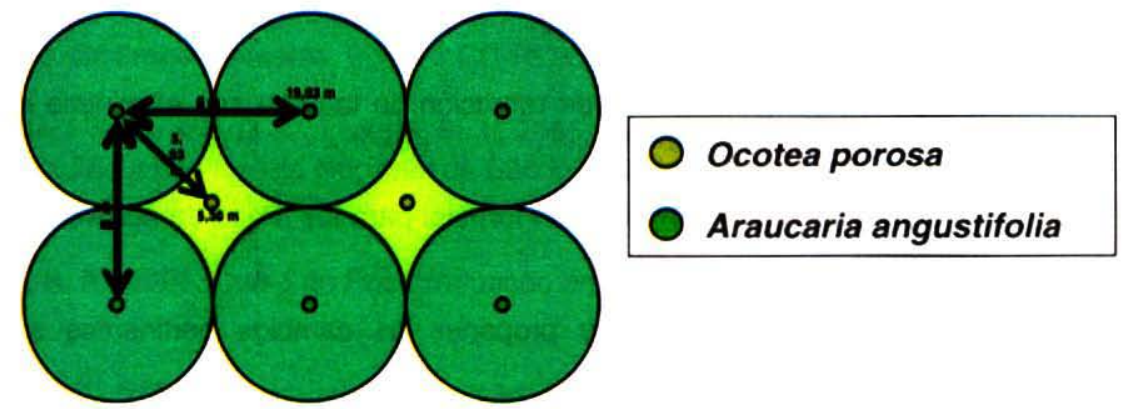

Figura $\mathrm{N}^{\circ} 7$

DISEÑO DEL ENSAYO DE RECUPERACIÓN DEL ÁREA ALTERADA POR FUEGO

Otra actividad de monitoreo está siendo desarrollada en un ensayo instalado el año 2004 dentro de una de las parcelas permanentes. Se trata de un plantio de aumento de densidad con Araucaria angustifolia, utilizando plántulas, realizado en la mitad de la superficie de la parcela, es decir, en $1250 \mathrm{~m}^{2}$, en filas regulares. Aún no se ha efectuado ninguna evaluación estadistica del experimento, pero las primeras observaciones muestran poco crecimiento de las plántulas y alta tasa de mortalidad por predación del mono. El objetivo es comparar la regeneración natural del pino-paraná con y sin técnicas de aumento de densidad, además de evaluar la relación costo-beneficio de la aplicación de dichas técnicas en el bosque con araucaria. También para este ensayo se utilizará el SIG como herramienta de monitoreamiento.

\section{CONCLUSIONES}

El desarrollo del presente trabajo tiene como objetivo final recuperar los bosques de esta Reserva, los cuales fueron afectados por un proceso histórico de explotación maderera, cuyo reflejo hoy se traduce en extensas áreas dominadas por taquara, que impiden el desarrollo de la dinámica natural de estos bosques. Sin embargo, y por sobre todo, este proyecto quiere poder demostrar que es factible el uso y la conservacion de estos valiosos bosques para el beneficio ambiental, social y económico de la sociedad brasileña.

Por lo tanto, este proyecto será ejecutado procurando la protección y uso comercial de los recursos forestales de la Reserva, considerándose las restricciones y las potencialidades del medio físico y biótico, asi como los requerimientos del propietario.

En sintesis, se espera poder formular y ejecutar el modelo de manejo forestal para un periodo de 5 años, según la siguiente secuencia de trabajos:

Determinar los diferentes usos de la Reserva (Zonificación)

Definir tipologias de vegetación según análisis fitosociológico y con apoyo de geo-procesamiento. 
Probar diferentes tratamientos silviculturales para las tipologias definidas y sus respectivos esquemas de manejo.

Evaluar diferentes métodos de remoción de taquara con el mínimo impacto para los remanecientes.

Establecer el plan de ordenación forestal para las áreas de aprovechamiento maderero.

Monitorear los resultados y proponer los cambios pertinentes si fuese necesario.

Evaluación social, ecológica y económica del plan de manejo forestal.

\section{AGRADECIMIENTOS}

Los autores desean agradecer el apoyo del profesor C.V. Roderjan de la UFPR, del equipo del Laboratorio de Ecologia de EMBRAPA Florestas y del Museo Botánico de Curitiba en la identificación botánica. También expresan su gratitud por el apoyo en el trabajo de terreno a las siguientes personas: Admilson Ruppel, Rafaelo Balbinot, Tiago Stepka, Fernando Luis Dlugosz y Klèber dos Santos.

\section{REFERENCIAS}

Cruz, P., 2005. Conferência sobre manejo florestal. In: Rosot et al. (Eds. Técnicos). Workshop "Aspectos teóricos e práticos do manejo florestal: Um enfoque para a Araucaria angustifolia". Curitiba, 3 e 4 de novembro de 2005. Conferência, palestras e resultados. Colombo : Embrapa Florestas. 1 CDROM.

Cruz, P. y Rothermel, H., 1998. Sistema simplificado de ordenación forestal para la pequeña y mediana propiedad. In: Primer Congreso Latinoamericano IUFRO, Valdivia, 1998. Proceedings ... Santiago : CONAF. p. 3.

Dlugosz, F. L., 2005. Classificação orientada a regiōes na discriminação de tipologias da floresta ombrófila mista usando imagens orbitais Ikonos. Curitiba, 2005. Dissertação (Mestrado em Engenharia Florestal) - Setor de Ciências Florestais, Universidade Federal do Paraná.

Guerra, M. P., 2000. Exploração, manejo e consenvação da araucária (Araucaria angustifolia). In: Simões, L.L.; Lino, C.F. (Eds) Sustentável Mata Atlântica: a exploração de seus recursos florestais. São Paulo: SENAC, p. 85-101.

Kurasz, G., 2005. Sistema de Informações Geográficas aplicado ao Zoneamento Ambiental da Reserva Florestal Embrapa/Epagri, Caçador-SC. Curitiba, 2005. 137 p. Dissertação (Mestrado em Ciências Florestais). Universidade Federal do Paraná.

Kurasz, G., Dlugosz, F. L., Rosot, N. C., Rosot, M. A. D., Oliveira, Y. M. M., 2004. Levantamento 
semidetalhado de solos para atualização de legenda na Reserva Florestal Embrapa/Epagri de Caçador-SC. In: Evento de Iniciação Cientifica da EMBRAPA Florestas, 3., Colombo. Anais... Colombo: Embrapa Florestas, 2004. 1 CD-ROM.

Narvaes, I. S., Brena, D. A., Longhi, S. J., 2005. Estrutura da regeneração natural em Floresta Ombrófila Mista na Floresta Nacional de São Francisco de Paula, RS. Ciência Florestal, Santa Maria, v. 15, n. 4, p. 331-342.

Rivera, H. A., 2006. Curso de Pós-graduação em engenharia florestal. Comunicação pessoal. 2006.

Rosot, N.C., 2005. Açōes de recuperação em área degradada por fogo em remanescente de floresta ombrófila mista: resultados parciais. In: Oliveira et al. (Eds.) Seminário sobre recuperação de áreas degradadas em floresta com araucária. Curitiba, 25 de outubro de 2005. Apresentações e resumos das palestras. Colombo : Embrapa Florestas, 1 CDROM.

Rothermel, H., 2002. Economia del manejo sustentable: Uma alternativa para el bosque nativo. Santiago: Ediciones Universidad Mayor, $178 \mathrm{p}$. 
